\title{
Investigation on clinical healthy swine carrier status of Streptococcus suis in Hebei Province of China
}

\author{
Ping Rui ${ }^{1}$, Zeng-Jun Ma ${ }^{1 *}$, Qiu-Yue Wang ${ }^{1}$, Xiang-zhai Zhang ${ }^{1}$, Jin-Xia Wang ${ }^{2}$, Yan-Ying \\ Zhang ${ }^{1}$ and Hai Fang ${ }^{1}$ \\ ${ }^{1}$ Key Laboratory of Preventive Veterinary Medicine in Hebei Province, Hebei Normal University of Science and \\ Technology, Qinhuangdao, 066004 China. \\ ${ }^{2}$ The Second Hospital of Qinhuangdao, Changli, 066600, China.
}

Accepted 25 July, 2012

\begin{abstract}
A total of 600 samples of nose swabs collected from Hebei Province, China, were examined by polymerase chain reaction (PCR) for the presence of Streptococcus suis in healthy swine and the serotype were identified. Results showed that 148 strains $(24.67 \%)$ of 600 tested were positive with $S$. suis, including 27 strains (18.24\%) were identified to be type 7, 24 strains (16.22\%) were type 2 and 20 strains $(13.51 \%)$ were type 9 . But serotypes of other 75 strains $(50.68 \%)$ were undetermined. To our knowledge, this is the first epidemiological investigation of $S$. suis in healthy swine from Hebei Province of China.
\end{abstract}

Key words: Streptococcus suis, polymerase chain reaction (PCR), serotype, epidemiological.

\section{INTRODUCTION}

Streptococcus suis is an important pathogenic bacteria hazard in modern swine industry. It can be divided into 35 serotypes (type 1 to 34 and 1/2) according to the differences in antigenic properties of polysaccharide capsular. S. suis serotype 2 is considered to be the most widely popular and highest pathogenicity isolated in both swine and humans, which is also an important zoonotic pathogen. It reported that slaughterhouse employees can be infected with $S$. suis and to be considered as an occupational disease (He et al., 2000; Hu et al., 2000). In recent years, $S$. suis outbreaks in many countries from Europe, Americas and Asian. It can not only cause huge losses to the world's swine industry, but also endangers with public health and safety (Staats et al., 1997; Touil et al., 1998; Torremorell et al., 1998). S. suis serotype 2 (SS2) was discovered firstly in Guangdong Province, China, in 1990. There were two large outbreaks of SS2, people had been found infected with SS2 died in Jiang su, Si chuan Province, in 1998 (Shen et al., 2000; Liu et al., 2005). In addition to SS2, SS1, SS7, SS9, etc are

${ }^{*}$ Corresponding author. E-mail: mzj6699@126.com. Tel: +860335-2039084. Fax: +86-0335-2039084. also important serotypes in pigs. In order to investigate the presence of $S$. suis in normal swine herds in Hebei Province, 600 nasal swabs were collected from Shijiazhuang, Xingtai, Zhangjiakou, Cangzhou, Tangshan, Qinhuangdao and other areas of Hebei Province in May 2009 to October 2009, and then detected by polymerase chain reaction (PCR). This study can provide information for epidemiological investigation of $S$. suis, and has great significance for further monitoring and effective prevention to $S$. suis.

\section{MATERIALS AND METHODS}

Strains and antisera

S. suis standard strain SS2 was provided by Professor Lu Chengping, Nanjing Agricultural University, China. Standard strains SS1, SS7, SS9 was donated by researcher Cai Xuehui, Harbin Veterinary Research Institute China. Standard antiserum of S. suis type 1, type 2, type 7, type 9, 1/2 and 14 types were taken from college of Veterinary Medicine, Nanjing Agricultural University.

\section{Medium and reagents}

Medium was purchased from Qingdao Haibo biotech companies. Brain heart infusion broth was purchased from Oxoid company, 
Table 1. Primers of $S$. suis.

\begin{tabular}{|c|c|c|c|c|}
\hline $\begin{array}{l}\text { Target } \\
\text { genes }\end{array}$ & Primers'sequences $\left(5^{\prime}\right.$ to $\left.3^{\prime}\right)$ & $\begin{array}{l}\text { Annealing temperature } \\
\left({ }^{\circ} \mathrm{C}\right)\end{array}$ & $\begin{array}{l}\text { Length } \\
\text { (bp) }\end{array}$ & References \\
\hline $\begin{array}{l}\text { gdh-1 } \\
\text { gdh-2 }\end{array}$ & $\begin{array}{l}\text { GCAGCGTATTCTGTCAAACG } \\
\text { CCATGGACAGATAAAGATGG }\end{array}$ & 55 & 689 & Okwumabua et al. (2003) \\
\hline $\begin{array}{l}\operatorname{cps} 11-1 \\
\operatorname{cps} 11-2\end{array}$ & $\begin{array}{l}\text { GGCGGTCTAGCAGATGCTCG } \\
\text { GCGAACTGTTAGCAATGAC }\end{array}$ & 55 & 441 & Smith et al. (1999) \\
\hline $\begin{array}{l}\text { Cps2J-1 } \\
\text { Cps2J-2 }\end{array}$ & $\begin{array}{l}\text { ATGTTTGGAATACGCAGAGCAAAGAT } \\
\text { CAACAAGGGCTATTAAAGATACCGC }\end{array}$ & 55 & 351 & Wang et al. (2009) \\
\hline $\begin{array}{l}\text { cps7H-1 } \\
\text { cps7H-2 }\end{array}$ & $\begin{array}{l}\text { AGCTCTAACACGAAATAAGGC } \\
\text { GTCAAACACCCTGGATAGCCG }\end{array}$ & 55 & 251 & Wang et al. (2009) \\
\hline $\begin{array}{l}\mathrm{cps} 9 \mathrm{H}-1 \\
\mathrm{cps} 9 \mathrm{H}-2\end{array}$ & $\begin{array}{l}\text { GGCTACATATAATGGAAGCCC } \\
\text { CCGAAGTATCTGGGCTACTG }\end{array}$ & 55 & 388 & Smith et al. (1999) \\
\hline
\end{tabular}

ExTaq polymerase (5U/L), dNTPs (2.5 mmol/L each), $10 \times$ PCR buffer (containing $\mathrm{MgCL}_{2}$ ), DNA Marker DL2000 were purchased from TaKaRa Company, bacterial genomic DNA extraction kit purchased from Tiangen Biotech(Beijing) CO.,LTD.

\section{Primers}

Five pairs of primers were designed according to the reference (Okwumabua et al., 2003; Smith et al., 1999; Wang et al., 2009) respectively and synthesized in Sangon Biotech (Shanghai) Co., LTD. The details of the primers were listed in Table 1.

\section{Sample collection}

600 nasal swabs were collected from healthy pigs (different growth stage) on farms of Hebei Province in China, such as Shijiazhuang, Xingtai, Zhangjiakou, Cangzhou, Tangshan and Qinhuangdao. Samples were collected and processed to refrigerated storage.

\section{Bacteria culture and identification}

$100 \mu \mathrm{l}$ samples of nasal swab were inoculated into $2 \mathrm{ml}$ of Streptococcus liquid selection medium (containing $15 \mu \mathrm{g} / \mathrm{ml}$ polymyxin B, $30 \mu \mathrm{g} / \mathrm{ml}$ nalidixic acid and $0.2 \mathrm{~g} / \mathrm{ml}$ crystal purple) for 18 to $24 \mathrm{~h}$ at $37^{\circ} \mathrm{C}, S$. suis was observed in Gram's method by a microscope.

\section{Preparation of the templates}

$1000 \mu \mathrm{l} \mathrm{Gram-positive} \mathrm{Streptococcus} \mathrm{culture} \mathrm{liquid} \mathrm{were} \mathrm{centrifuged}$ at $10000 \mathrm{r} / \mathrm{min}$ for $1 \mathrm{~min}$, supernatant was discarded, resuspended

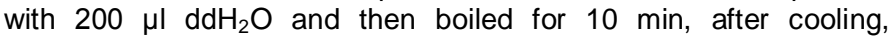
centrifuged at $7000 \mathrm{r} / \mathrm{min}$ for $5 \mathrm{~min}$, supernatant were stored at $20^{\circ} \mathrm{C}$. DNA was extracted using Bacteria genomic DNA extraction kit.

\section{PCR identification of S. suis}

Firstly, GDH sequence of $S$. suis was applied to identify the strain.
Samples were also identified by PCR based on the $S$. suis serotype $1,2,7,9$. The final PCR volume was $25 \mu \mathrm{l}$, the reaction components are as follows: $10 \times$ PCR bufferr ( $\mathrm{Mg}^{2+}$ Plus) $2.5 \mu \mathrm{l}$, dNTPs Mixture $(2.5 \mathrm{mM}) 2.0 \mu \mathrm{l}$, upstream and downstream primer $1.0 \mu \mathrm{l}$, Ex Taq DNA polymerase $0.2 \mu \mathrm{l}(5 \mathrm{U})$, DNA template $2.5 \mu \mathrm{l}$, added $\mathrm{dd}_{2} \mathrm{O}$ to $25 \mu \mathrm{l}$. PCRs consisted of 30 cycles of denaturation for $5 \mathrm{~min}$ at $95^{\circ} \mathrm{C}$, then $95^{\circ} \mathrm{C}$ denaturation $15 \mathrm{~S}$ (gdh and $\mathrm{cps} 1 \mathrm{I}$ ) or $94^{\circ} \mathrm{C}$ for $30 \mathrm{~s}$ (Cps2J, Cps7H, Cps9H), annealing at $55^{\circ} \mathrm{C}$ for $45 \mathrm{~s}$, and extension for $30 \mathrm{~s}$ at $72^{\circ} \mathrm{C}$. A final extension was performed for $10 \mathrm{~min}$ at $72^{\circ} \mathrm{C}$. PCR reaction condition of the rest genes are the same as except for annealing temperature. Simultaneously, negative control was designed. The PCR products were detected by electrophoresis in $1.2 \%$ agarose gel.

\section{Isolation and identification of S. suis}

The strain was identified as S. suis in morphology. S. suis is Grampositive cocci in pairs or short chains of broth cultures by microscopy. Bacterial liquid rules on blood agar plates, at $37^{\circ} \mathrm{C}$ for $18 \sim 24 \mathrm{~h}, 3 \sim 6$ colonies each plate with a hemolytic, smooth, moist, white translucent, diameter $1 \sim 2 \mathrm{~mm}$ were picked selectively, and inoculated into $2 \mathrm{ml} 5 \%$ bovine serum of THB, suspected of $S$. suis were stored at $4^{\circ} \mathrm{C}$. Finally, PCR methods established above were used to identified the isolated bacteria.

\section{Serum agglutination test}

Each drop of diagnose serum antibodies known and bacilli were mixed in the slide, a few minutes after, it was identified as positive when there was emergence of visible agglutination. Also set up normal saline as control. PCR identification as $S$. suis serotype 2 were to have further identification with $1 / 2$ Standard antiserum, PCR identification as $S$. suis serotype 1 , and $S$. suis serotype 14 and $1 / 2$ standard anti-serum were used for further identification, respectively.

\section{RESULTS}

\section{S. suis carrying in different regions}

PCR analysis showed that 148 samples in 600 nasal 
Table 2. Results of $S$. suis positive rates.

\begin{tabular}{lccc}
\hline Regions & Samples number & SS positive number & Positive rate (\%) \\
\hline Qinhuangdao & 100 & 30 & 30 \\
Tangsan & 100 & 32 & 32 \\
Xingtai & 100 & 25 & 25 \\
Shijiazhuang & 100 & 34 & 34 \\
Zhangjiakou & 100 & 11 & 11 \\
Cangzhou & 100 & 17 & 17 \\
Total & 600 & 148 & 24.67 \\
\hline
\end{tabular}

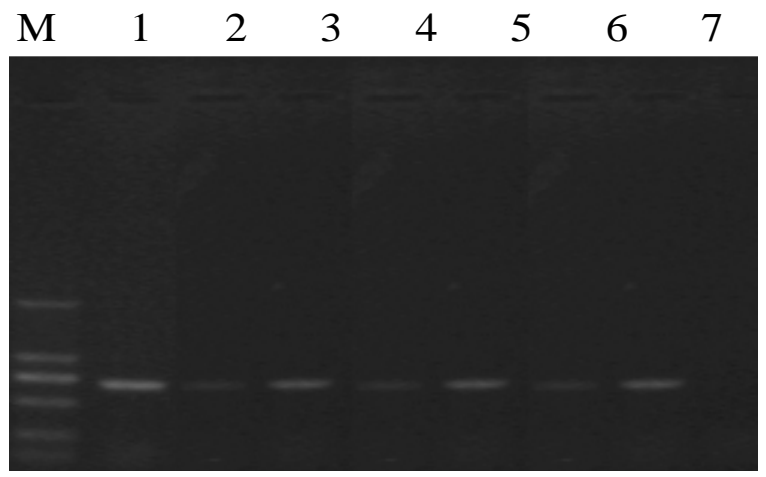

Figure 1. PCR result of gdh gene from $S$. suis. M: DS2000 Maker; 1-7, gdh positive strain; 8, negative control.

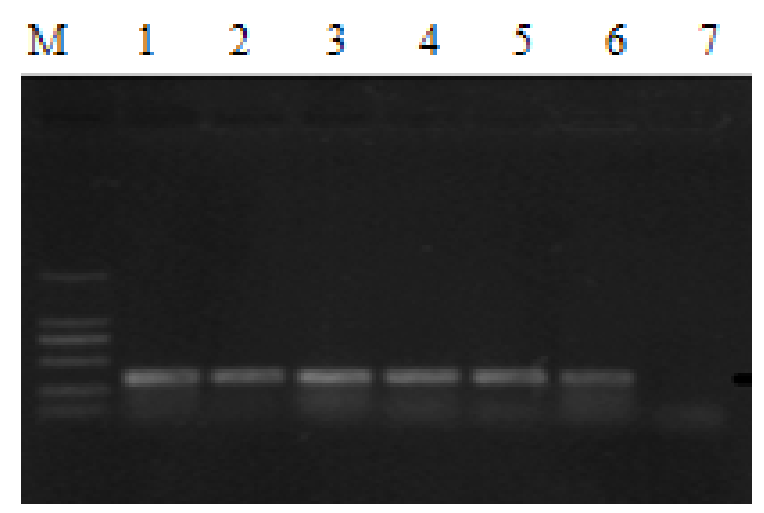

Figure 2. PCR result of cps2J gene from $S$. suis $\mathrm{M}$ : DS2000 Maker; 1-6, cps2J positive strain; 7, negative control.

swabs were positive for $S$. suis, the positive rate was $24.67 \%$, results were shown in Table 2. Zhangjiakou, Cangzhou positive rates were 11 and $17 \%$, significantly lower than other regions, the difference was significant $(p<0.01)$, Qinhuangdao, Tangshan, Shijiazhuang positive samples were high, were 30,32 and $34 \%$, significantly higher than other regions, which showed that the $S$. suis infection had some regional differences (Table 2). The electrophoresis graphs of gdh, cps2J, cps1l, cps7H and
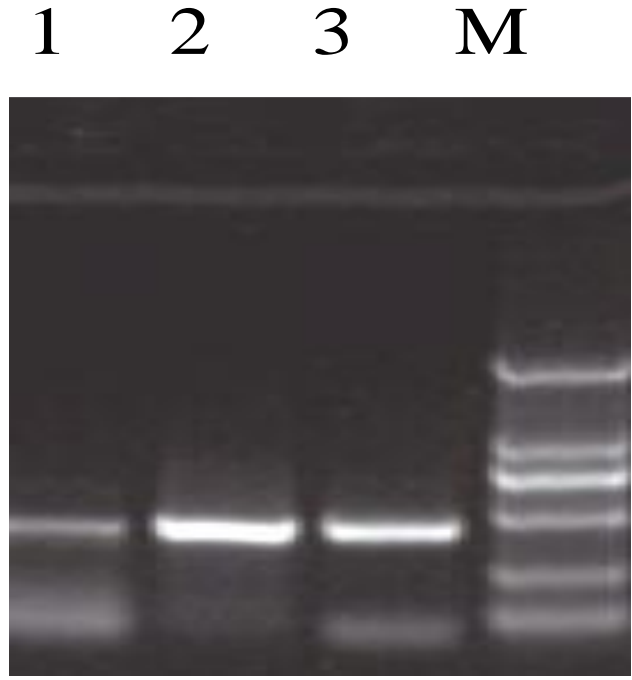

Figure 3. PCR result of cps1l gene from S.suis M: DS2000 Maker; 1-2, cps1l positive strain; 3, positive control.
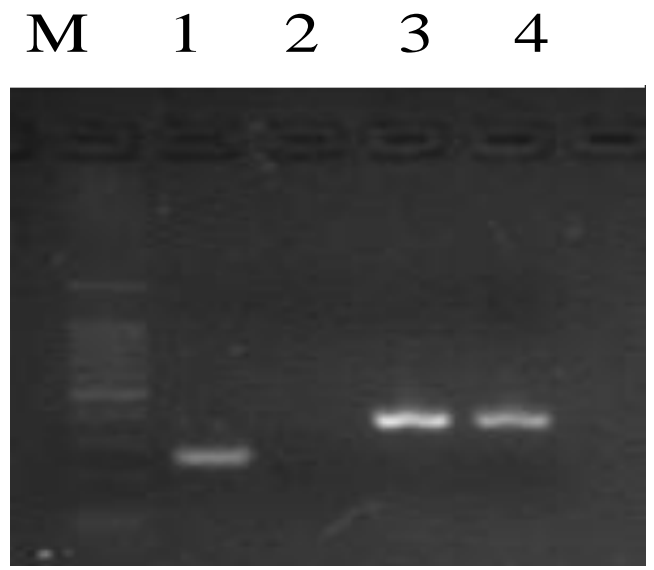

Figure 4. $\mathrm{PCR}$ result of $\mathrm{cps} 7 \mathrm{H}$ and $\mathrm{cps} 9 \mathrm{H}$ gene from $S$. suis $\mathrm{M}$ : 100 bp DNA ladder;1, cps7H positive strain; 3-4, cps9H positive strain.

cps9H genes of some strains respectively are shown as Figures 1, 2, 3 and 4. 
Table 3. Major pathogenic $S$. suis serotype carrying cases in different regions.

\begin{tabular}{lcccccc}
\hline \multirow{2}{*}{ Region } & $\begin{array}{c}\text { Total sample } \\
\text { number }\end{array}$ & $\begin{array}{c}\text { SS1 } \\
\mathbf{n}(\%)\end{array}$ & $\begin{array}{c}\text { SS2 } \\
\mathbf{n}(\%)\end{array}$ & $\begin{array}{c}\text { SS7 } \\
\mathbf{n}(\%)\end{array}$ & $\begin{array}{c}\text { SS9 } \\
\mathbf{n}(\%)\end{array}$ & $\begin{array}{c}\text { others } \\
\mathbf{n}(\%)\end{array}$ \\
\hline Qinhuangdao & 100 & $1(1.0)$ & $6(6)$ & $4(4)$ & $1(1)$ & $18(18)$ \\
Tangshan & 100 & 0 & $5(5)$ & $5(5)$ & $10(10)$ & $12(12)$ \\
Xingtai & 100 & 0 & $4(4)$ & $1(1)$ & $6(6)$ & $14(14)$ \\
Shijiazhuang & 100 & 0 & $4(4)$ & $12(12)$ & $4(4)$ & $14(14)$ \\
Zhangjiakou & 100 & 0 & $1(1)$ & $2(2)$ & 0 & $8(8)$ \\
Cangzhou & 100 & $1(1)$ & $4(4)$ & $3(3)$ & 0 & $9(9)$ \\
Total & 600 & $2(0.33)$ & $24(4)$ & $27(4.5)$ & $20(3.3)$ & $75(12.5))$ \\
\hline
\end{tabular}

Table 4. Different $S$. suis serotypes mixed infection.

\begin{tabular}{ccccccc}
\hline \multirow{2}{*}{$\begin{array}{c}\text { Total sample } \\
\text { number }\end{array}$} & $\begin{array}{c}\text { SS positive sample } \\
\text { number }\end{array}$ & $\begin{array}{c}\text { SS2+SS7 } \\
\mathbf{n}(\%)\end{array}$ & $\begin{array}{c}\text { SS2+SS9 } \\
\mathbf{n}(\%)\end{array}$ & $\begin{array}{c}\text { SS7+SS9 } \\
\mathbf{n}(\%)\end{array}$ & $\begin{array}{c}\text { SS2+SS7+SS9 } \\
\mathbf{n}(\%)\end{array}$ & $\begin{array}{c}\text { SS1+ SS2 } \\
\mathbf{n}(\%)\end{array}$ \\
\hline 600 & 148 & $6(1)$ & $4(0.67)$ & $10(1.67)$ & $3(0.5)$ & $1(0.17)$ \\
\hline
\end{tabular}

\section{Major pathogenic S. suis serotype carrying cases in different regions}

PCR test results showed that each serotype carrying case as follows: SS7 were the highest (4.5\%), followed by SS2 up to $4 \%$; SS9 of 3.3\%; SS1 was the least, only $0.33 \%$. The positive rate of SS7 in Shijiazhuang or Qinhuangdao was significantly $(p<0.01)$ higher than other areas; SS1 positive rate was lower, in addition to one was detected in Qinhuangdao, Cangzhou, other regions were not detected positive. The positive rate of SS9 in Qinhuangdao was significantly higher than other regions $(p<0.01)$ (Table 3).

\section{S. suis serotype mixed infections in different parts}

Statistics of nasal swab test results showed that the same one sample could be infected with two or more different serotypes of Streptococcus suis. But the samples were limited, the proportion in the sample was less than 5\%. The samples of infected with both SS7 and SS9 sample were the most, up to $1.67 \%$ of the total sample, both SS2 and SS7 were the second, accounting for $1 \%$, both SS2 and SS9 infection accounted for $0.67 \%$. There were also infected with SS2, SS7 and SS9 or more serotypes, but the proportion was very small, only $0.5 \%$. (Table 4).

\section{Bacterial isolatation and PCR results}

Gram-positive cocci in pairs or short chains of 528 broth cultures by microscopic examination were isolated, 61
S. suis strains were obtained, isolated rate was $11.55 \%$. SS2 and SS7 were the most, each 11 stains was $(2.08 \%)$. SS9 were $8(1.52 \%)$, SS1 was not isolated. Nofinalized SS were 31 stains $(5.87 \%)$.

\section{Serum agglutination test}

Results for 30 isolates with a slide serum agglutination test showed that $11 S$. suis serotype 2 bacilli only had antiserum agglutination with $S$. suis type 2 , not with $S$. suis $1 / 2$ type, so it was judged as $S$. suis type 2 . S. suis serotype 7 (11) and type 9 (8) bacilli had specific agglutination with $S$. suis serotype 7, type 9 antiserum respectively. All results were consistent with the PCR analysis results.

\section{DISCUSSION}

The $S$. suis detection rate is different in the normal swine herds in different regions of China. Yang et al. (2009) reported 14 strains of $S$. suis in 248 pig tonsils were detected collected from 20 different regions of China, $S$. suis isolated from the tonsils were obtained from southern region of China, which may be related to $S$. suis outbreak and its hot and humid climate in south. Lu et al. (2008) isolated a highly pathogenic $S$. suis type 2 containing eight major virulence factor from 40 tonsil collected from a slaughterhouse, Jiangsu Province. Luo et al. (2009) investigated the carrying $S$. suis of healthy pigs from nasal swabs, throat swabs and tonsil in Ziyang, S. suis type 2 was only detected, S. suis carrier rate was $14.93 \%$, concentrated in July, has a more obvious 
seasonal characteristics. However, there were no related reports about carrying $S$. suis cases in clinical healthy pig herds in Hebei Province. In this study, 600 healthy pigs nasal swabs were first detected by PCR and isolate collected from 6 different regions of Hebei Province, 148 samples were found to be SS-positive $(24.67 \%)$, mainly prevalent serotypes of $S$. suis type 1 , type 2 , type 7 , type 9 , total 73 , other types were 75 , was lower than $S$. suis detected in Heilongjiang (29\%), Jilin (27\%), Liaoning (34\%) Province, report by Shu-Jie Wang et al. (2009). It confirmed that $S$. suis is also widespread in Hebei Health pig herds, and the coexistence of multiple serotypes, indicating that pigs carrying $S$. suis serotype is complex and diverse in normal pigs farms.

Study found that SS7 had the highest detection rate, $4.5 \%$, followed by SS2, SS9, and the detection rates were 4 and $3.3 \%$ respectively, these results suggested SS2, SS7 and SS9 were the most important popular serotypes in the Hebei region of China, and SS2 was zoonotic disease. More attention should be paid to this disease. SS7 was the highest detection strain. The authors found the pig cases infected with SS7 in Hebei, therefore, it needs to conduct deeper research on SS7 and strengthen prevention and control. SS1 carrier rate was relatively low $(0.33 \%)$. There were multiple $S$. suis serotypes in the same nasal swabs by PCR, but it was small rate related to the carrier rate of each serotypes, its epidemiological significance remains to be studied. The study also found that, samples collected from Zhangjiakou, Cangzhou was significantly lower than other places of the province, can be inferred there are some regional differences in SS infection or the incidence of different farms will be different.

PCR is the most commonly method for the detection of S. suis. But PCR cannot distinguish between $S$. suis type 1 and type $14, S$. suis $1 / 2$ and type 1 , type 2 . To make test results more accurate, standard antiserum of $S$. suis type 2, type 7 , type 9,14 and $1 / 2$ prepared by our laboratory were used to have a re-examination for this epidemiological survey. $S$. suis type 1 and $1 / 2$ were not isolated in this study, it may be relative to the two serotypes little in Hebei Province.

\section{ACKNOWLEDGEMENT}

This study was financially supported by grant from Natural funded project of Hebei (No.C2009000877), Education Department of Hebei Province (No. 2008448).

\section{REFERENCES}

He JH, Xu JX, Hou JB, Qian JF, Wang JC, Liu DX, Zhu CG, Xing JC, Zhang ZJ, Lu JG, Gao SW (2000). One cases pigs of outbreak and epidemiology of Acute septicemia caused by streptococcus suis II . Chin. J. Zoonoses 16(I):109.

Hu XS, Zhu FC, Wang H, Chen SY, Wang GH, Sun JZ, Hua CT, Yang HF (2000). Studies on human streptococcal infectious syndrome caused by infected pigs. Chin. J. Prev. Med. 34(3):150-152.

Liu HL, Zhao YG, Wang JW, Zhao YL, Li L, Zheng DX, Sun CY, Wang S, Liu PL, Song CP , Fan WX, Chen YP, Wang ZL (2005). Isolation and identification of pathogen related to recent outbreak of pighuman infection in Sichuan province. Chin. J. Microbiol. Immunol. 25(12):1006-1010.

Lu LX, He KW, Ni YX, Zhang XH, Yu ZY, Zhou JM (2008). Isolation and identification of virulent Streptococcus suis type 2 fromtonsillar specimens of slaughtered healthy pigs. Chin. J. Zoonoses 24(4):379383

Luo LZ, Wang X, Cui ZG, Li YC, Guo ZQ, Jin D, Zheng H, He SS, Liu $X C$, Jia $Y$, Liao $A B$, Jing $H Q$ (2009). Isolation of Streptococcus suis type 2 from healthy pigs in Ziyang district of Sichuan province and analysis of their molecular characteristics. Chin. J. Zoonoses 25(9):842-845.

Okwumabua O, O'Connor M, Shull E (2003). A polymerase ehain reaction (PCR) assay specific for Streptococcus suis based on the gene encoding the glutamate delydrogenase [J]. FEMS Microbiol. Lett. 218:79-84.

Shen J, Sun JZ, You YM, Bo YJ, Wang CL, Zang L, Chen SY (2000). Analysis of prevention and control effect on Human Streptococcal Infectious Syndrome Caused by Infected Pigs in Rugao city. J. Math. Med. 13(3):257-258

Smith HE, Veenbergen V, van der Velde J, Damman M, Wisslink HJ, Smits MA (1999). The cps genes of Streptococcus suis serotypes 1 , 2, and 9: development of rapid serotype-specific PCR assays [J]. J. Clin. Microbiol. 37:3146-3152.

Staats JJ, Feder I, Okwumabua O, Chengappa MM (1997). Streptococcus suis past and present. Vet. Res. Commun. 21(6):381 407.

Torremorell M, Calsamiglia M, Pijoan C (1998). Colonization of sucking pigs by Streptococcus suis with particular reference to pathogenic sereotype 2 strains. Can. J. Vet. Res. 62(I):21-26.

Touil F, Higgins R, Nadeau M (1988). Isolation of Streptococcus suis from diseased pigs in Canada. Vet. Microbiol. 17(2):171-177.

Wang SJ, Lei LC, Xu M, Sun CJ, Li CJ, Cai XH, Liu YG, Zhang Q, Liu DQ, Shi WD (2009). Isolation, identifiation and epidemiological analysis of swine Streptococcus in the northeast region of China. Chin. J. Vet. Sci. 29(7):877-881.

Yang Z, Wang KC, Fan WX, Jiang P (2009). Epidemiological investigation on the carrier status of Streptococcus suis in healthy pigs. Chin. J. Zoonoses 25(10):977-979. 